\title{
MEJORAR LA EDUCACIÓN ARTÍSTICA 12-16: ESTABLECER VÍNCULOS ENTRE TEORÍA Y PRÁCTICA
}

MELHORAR A EDUCAÇÃO ARTÍSTICA 12-16: ESTABELECER VÍNCULOS ENTRE A TEORIA E A PRÁTICA

IMPROVING ART EDUCATION: 12-16 ESTABLISHING LINKS BETWEEN THEORY AND PRACTICE

\section{ESTHER COLLADOS CARDONA}

Universitat Autònoma de Barcelona

Tipo de artigo: Original

\section{RESUMEN}

En los niveles de educación obligatoria, la Educación Artística ha sido y a menudo todavía es un área desatendida donde faltan recursos y estrategias, o bien es necesario optimizar las ya existentes. Su complejo carácter, provoca que a menudo se desconozca e infravalore su capacidad educadora, ignorando su contribución en la formación integral de las personas.

Las teorías y los paradigmas que nos hablan de la Educación Artística, ponen sobre la mesa reflexiones, marcos de referencia atractivos y estimulantes que nos inspiran, ayudan y orientan a definir los grandes objetivos de lo que debe ser la educación en el siglo XXI, pero descuidan el desarrollo de aspectos más aplicativos, que se traduzcan en programas de mejora en la escuela, olvidando la realidad de lo que acontece en su llamada "caja negra".

Partiendo de la necesidad de establecer vínculos entre teoría y práctica, nació la pregunta central que se formuló el proyecto "Análisis y experimentación de actuaciones para la mejora de la Educación Artística en la escuela secundaria obligatoria":

¿Que factores pueden introducir mejoras en el aprendizaje de las artes plásticas y visuales en la escuela secundaria obligatoria?

De las que se derivan otras de no menos importantes:

¿Cual queremos que sea el papel de la educación artística en la formación básica de los ciudadanos? ¿Quién debe formular el conocimiento deseable para nuestros alumnos? ¿Que modelo de educación artística deseamos desarrollar en nuestras aulas? ¿Que tipo de conocimientos debería priorizar este modelo?

Las razones para plantear esta pregunta parten de la necesidad de dinamizar la práctica educativa que se desarrolla en este campo, revisando su actualidad y pertinencia en los nuevos escenarios que hoy se dibujan en nuestros centros educativos, y que son reflejo de profundos cambios en un panorama social que se revela complejo y que plantea retos a las nuevas sociedades pluriculturales que se están fraguando. 


\section{RESUMO:}

Na escolaridade obrigatória, a educação artística ainda é muitas vezes negligenciada, sendo uma área onde faltam recursos e estratégias, ou onde é necessário optimizar os já existentes. A sua natureza complexa, propicia a que muitas vezes se desconheça e se subestime a sua capacidade educadora, ignorando a sua contribuição para a formação integral dos indivíduos.

As teorias e paradigmas que conhecemos sobre educação artística, colocam-nos sobre a mesa reflexões, marcos de referência atractivos e estimulantes que nos inspiram, ajudam e orientam a definir os principais objectivos da educação no século XXI, mas negligenciando o desenvolvimento de aspectos mais práticos, que resultem na melhoria dos programas escolares, ignorando a realidade do que acontece na sua chamada "caixa negra".

Com base na necessidade de estabelecer ligações entre a teoria e a prática, foi formulada a questão central do projecto "Análise e experimentação de acções para a melhoria da educação artística obrigatória no ensino secundário":

Quais os factores que podem introduzir melhorias na aprendizagem das artes visuais no ensino secundário obrigatório?

Desta pergunta derivam de outras questões não menos importantes:

Qual queremos que seja o papel da educação artística na formação básica dos cidadãos? Quem deve formular o conhecimento desejado para os nossos alunos? Que modelo de educação artística queremos desenvolver nas nossas escolas? Que tipo de conhecimento deve privilegiar esse modelo?

As razões para levantar esta questão partem da necessidade de revitalizar a prática educativa que se desenvolve nesta área, revendo a sua actualidade e relevância nos novos cenários que hoje se desenham nas nossas escolas, e que são o reflexo de profundas mudanças num panorama social que se revela complexo, onde são colocados novos desafios para sociedades multiculturais emergentes.

\section{ABSTRACT}

In the levels of compulsory education, art education is still often a neglected area where there is a lack of resources and strategies, or where it is necessary to optimize the existing ones. Its complex nature often makes its capacity for education unknown and undervalued, ignoring its contribution to the integral formation of individuals.

The theories and paradigms that tell us about Art Education expose ideas and appealing and stimulating frameworks that inspire us, help us guide and define the main objectives of what education in the twenty-first century must be, but they neglect the development of more applicable aspects, which result in impro- 
vement programs in schools, ignoring the reality of what happens in its so-called "black box".

Based on the need to establish links between theory and practice, the central question which was formulated in the project "Analysis and experimentation of actions for the improvement of Art Education in compulsory secondary school" was born:

What factors can make improvements in the learning of visual and plastic arts in secondary compulsory school?

From which others not less important derive:

What role do we want arts education to have in the basic training of citizens? Who should formulate the desired knowledge for our students? What model of arts education do we wish to develop in our classrooms? What kind of knowledge should this model prioritize?

The reasons for raising this question are based on the need to revitalize the educational practice that is developed in this area, and revise its current relevance in the new settings that are drawn today in our schools, and that reflect the deep changes in a social scene which reveals itself complicated and which poses new challenges for the multicultural societies that are brewing.

PALABRAS CLAVE: educación artística, prácticas educativas, educación visual y plástica, metodologías didácticas, didáctica artes plásticas.

PALAVRAS-CHAVE: Educação Artística, práticas pedagógicas, educação visual e plástica, metodologias pedagógicas, ensino das artes visuais.

KEYWORDS: art education, educational practices, visual and plastic education, teaching methodologies, teaching plastic arts.

\section{EL PUNTO DE PARTIDA}

Si aceptamos la premisa de que la educación artística representa más que otras materias reflejo de los valores de una sociedad (GARDNER, 1994), es fácil adivinar que la nuestra no le otorga un papel nada relevante ni significativo, que se traduce en su escasa presencia y visibilidad en los niveles educativos correspondientes a la educación obligatoria, producto entre otras razones del desconocimiento y la infravaloración de su capacidad educadora.

El desinterés por la educación artística que manifiesta la sociedad española no parece nada nuevo (ARAÑó, 1989), hecho al que debemos añadir los sucesivos cambios curriculares generados por los continuos vaivenes en las legislaciones educativas y su poca visibilidad y tímida presencia en los estudios superiores, a la que nada favorece la indefinición epistemológica en que se halla sumida el área (AGIRRE, 2005).

Este panorama, algo desolador sin duda, no significa que no se estén produciendo experiencias interesantes en relación al arte y a la educación, pero si que más a menudo de lo que sería conveniente, se trata de experiencias singulares y difícilmente extensibles a la mayor parte de 
nuestros centros educativos, ya que lamentablemente en el marco de la educación obligatoria la educación artística aún es considerada un lujo innecesario, que ha sido y a menudo todavía es un área desatendida donde faltan recursos y estrategias, o donde es necesario optimizar las ya existentes.

Caracterizada por su considerable complejidad, a menudo se desconoce e infravalora su capacidad educadora, ignorando su contribución en la formación integral de las personas.

Las teorías y los paradigmas que nos hablan de la educación artística, ponen sobre la mesa reflexiones, grandes marcos de referencia de indudable e indiscutible interés que nos inspiran, ayudan y orientan a situar los grandes objetivos de lo que debe ser la educación en el siglo XXI, pero un buen docente "no puede separar su conocimiento teórico de su conocimiento práctico, ya que para construir el conocimiento profesional necesita tener experiencias que aporten significado y pongan a prueba la teoría, a su vez que la teoría educativa ilumina, dirige y corrige la acción. Realmente la teoría educativa sin práctica es pura especulación porque nos habla de problemas abstractos, que no tienen sentido y que no son los que tenemos. Ahora bien, la práctica sin teoría es repetitiva y divagante." (BENEJAM, 2004)

La teoría y la reflexión se revelan como herramientas indiscutibles e indispensables en momentos de cambio, pero únicamente si van seguidas de la acción, que tiene por finalidad materializar y experimentar estas. Partiendo de la necesidad de establecer vínculos entre teoría y práctica, nació la pregunta central que se formula este trabajo:

$¿$ Que factores pueden introducir mejoras en el aprendizaje de las artes plásticas y visuales en la escuela secundaria obligatoria?

Las razones y motivaciones para plantear esta pregunta parten del interés por mejorar mi trabajo diario con adolescentes, en mi convencimiento de que la educación artística no debería ser un obstáculo más que salvar en la escuela, sino una herramienta que les ayudara a transitar por la vida, y que les proporcionara la ocasión de vivir el arte como una experiencia enriquecedora, (DEWEY, 1949; AGIRRE, 2005) que les aporte herramientas para comprender y comprenderse.

\section{EL PLANTEAMIENTO DE LA INVESTIGACIÓN: OBJE- TIVOS Y METODOLOGIA}

El proyecto se planteó como finalidad el análisis y la experimentación de situaciones de aprendizaje en relación a las artes visuales en contextos específicos, buscando metodologías eficaces y prácticas educativas enriquecedoras y viables de ser transferibles a otros contextos educativos y que en consecuencia conduzcan a la mejora de la educación artística en la escuela.

Este proyecto también se planteó impulsar la reflexión y el debate entre los docentes de la disciplina, situando la reflexión sobre su propia práctica como punto de partida para construir conocimiento profesional, y cooperación entre iguales.

Es importante destacar que este proyecto, concebido como piloto, no pretende producir cambios en las prácticas escolares de fuera-adentro (COCHRAN; SMITH; LYTLE, 2002), sino impulsar y promover procesos de reflexión sobre el sentido de nuestras prácticas y de sus efectos en los aprendizajes, identificando a que concepciones docentes responde y produciendo conocimiento profesional que se convierta en el motor que transforme nuestras acciones y en consecuencia, modifique nuestra práctica.

Los objetivos más destacables en que se concretó el proyecto fueron los siguientes:

Observar y analizar la situación de la educación artística en relación a como se producen sus aprendizajes en tres instituciones educativas.

Diseñar y experimentar situaciones de aprendizaje que mejoraran la comprensión del hecho artístico, proporcionando las orientaciones, recursos y estrategias metodológicas necesarias para poder ser desarrolladas en el 
marco del currículum vigente de educación visual y plástica para los niveles de secundaria obligatoria.

Generar espacios de reflexión sobre la práctica docente que impulsen y promuevan el trabajo cooperativo entre docentes de secundaria.

Analizar los resultados obtenidos y extraer conclusiones acerca de los factores que puedan contribuir a mejorar el aprendizaje de las artes plásticas en los niveles de la secundaria obligatoria.

Elaborar un catálogo de posibles problemas que la aplicación de las nuevas propuestas puedan generar en el contexto educativo.

Este trabajo se desarrolló a partir de la construcción y estudio de tres casos en interacción con los centros educativos y los profesionales que participaron, hecho que posibilitó la "experimentación curricular" (ESCUDERO GÓMEZ, 2006) en entornos y contextos concretos desvelando las posibilidades de aprendizaje que podían generar.

El proyecto, que se desarrolló durante el curso escolar 2006-2007, se organizó y planificó en las tres fases diferenciadas, que se describen a continuación:

\section{Primera fase}

La primera fase se desarrolló durante el primer trimestre escolar y se propuso como objetivo observar, describir y analizar de que forma se desarrollaba el currículo de educación visual y plástica en las tres instituciones escolares que participaban, tratando de observar que tipo de prácticas escolares desarrollaban, con que clase de materiales curriculares trabajaban, como organizaban la programación, en que condiciones se impartía la docencia y a que concepciones respondía esta.

Los instrumentos de investigación que se utilizaron fueron entrevistas en profundidad con los profesores, análisis de lo materiales curriculares (libros de texto, materiales audio-visuales, programaciones...) y descripción de las condiciones en que se impartía la docencia.

\section{Segunda fase}

La segunda fase de la investigación se desarrolló durante los dos meses siguientes. En ella se propuso diseñar y planificar en colaboración con los docentes, nuevas situaciones de aprendizaje que plantearan nuevas formas de abordar el trabajo en el aula, que experimentaran con nuevas metodologías para desarrollar el currículum, con la finalidad de observar su eficacia en la transferencia de los aprendizajes y que el diseño de estos nuevos proyectos posibilitaran situaciones de aprendizaje compartido que impulsaran la reflexión sobre la práctica entre los docentes participantes.

\section{Tercera fase}

En la tercera fase se llevó a cabo la experimentación de las nuevas propuestas en el aula, y el relato de su desarrollo de forma densa y detallada, desvelando los posibles problemas y reflexiones que su puesta en marcha pudieran hacer emerger, así como su posterior evaluación en relación a los aprendizajes producidos y al grado de satisfacción y motivación de los agentes educativos implicados. Esta última etapa fue la más extensa, con una dedicación aproximada de seis meses.

\section{3- EL DESARROLLO DE LA INVESTIGACIÓN}

La primera fase consistió en observar como se desarrollaba la educación artística en cada uno de los contextos. Para ello se analizaron los materiales de aprendizaje, las programaciones, se entrevistó a los profesores, se observó el desarrollo de algunas clases, se visitaron las instalaciones y finalmente se redactó un informe, en el que se recogía el análisis de toda esta información, a partir del cual se propuso a los docentes diseñar proyectos de trabajo que aglutinaran una parte del currículo que tenían previsto desarrollar durante las siguientes semanas. El reto consistía en lograr incrementar el sentido de los aprendizajes que se producen en las aulas, encontrando estrategias que dinamizaran los procesos de aprendizaje. 
El proceso de trabajo que se desarrolló para diseñar los nuevos planes de trabajo fue enriquecedor y significó una oportunidad de aprendizaje para todos los docentes participantes. Se desarrollaron tres proyectos diferentes para cada una de las escuelas, en función del nivel educativo, de la situación del currículo y de las características y contexto del centro. Finalmente, quedaron formalizados en los siguientes:

1. “¿Hacemos un libro de artista?”: Una experiencia entorno a la práctica de la pintura como vehiculo de expresión y comunicación. ( Primer ciclo de ESO: 12-13 años)

2. “Creatividad y Cooperación: Un caso práctico de aprendizaje por problemas (PBL) aplicado al diseño gráfico." (Segundo ciclo de ESO: 14-15 años)

3. “Identidades." Un proyecto que articula una parte de los contenidos del currículo entorno al autorretrato. (Segundo ciclo de ESO: 14-15 años)

Se acotó la extensión temporal de cada uno de los proyectos en diez semanas aproximadamente, y fue desarrollado con una media de 70 alumnos.

El desarrollo exhaustivo de cada uno de estos proyectos no está dentro de los objetivos y posibilidades de este artículo, que se limitará a presentar sintéticamente cada uno de ellos.

\section{PRIMER PROYECTO: “HACEMOS UN LIBRO DE ARTISTA?”}

Una experiencia entorno a la práctica de la pintura como vehículo de expresión y comunicación.

Este proyecto de trabajo se desarrolló en las aulas correspondientes al segundo curso del Primer Ciclo de Educación Secundaria Obligatoria, que corresponde a la edad de 13 años.

El principal objetivo que se proponía era articular la parte del currículo correspondiente al conocimiento del color. Para ello se partió de la acción. La praxis como punto de partida y eje central, más adelante ya vendría la reflexión generada por su propia producción.
A continuación, se plantearon algunas propuestas para familiarizarse con la pintura y trabajar aspectos técnicos relativos al color. Una breve presentación de la profesora, invitó a todos los estudiantes a experimentar con el color e investigar con nuevas formas de aplicarlo en el soporte. Podían incorporar texturas, hacer sus propias pinturas con pigmentos... experimentando con las múltiples posibilidades que ofrece la práctica de la pintura y que pretendían aproximarlos a experimentar con el tipo de conocimientos que genera la praxis artística.

A continuación, se inició un diálogo en torno a sus conocimientos, experiencias y vivencias entorno al color y a las relaciones y analogías que establecemos en nuestro hacer diario. Se les propuso una asociación espontánea de imagen-palabra, para iniciar con ello un proceso de introspección que les pusiera en contacto con sus emociones, haciéndoles conscientes de cómo estas se generan, y de cómo el arte ofrece un espacio y un lenguaje para explorarlas.

Fue en esta fase donde se presentó el trabajo de algunos artistas, seleccionados por la proximidad formal de sus propuestas al trabajo que estaban realizando los estudiantes. Se dedicó una sesión para presentar sus obras, y se formularon cuestiones entorno al significado del arte y al sentido de su práctica.

El objetivo no era presentar respuestas, discursos cerrados entorno a aquello que debe ser el arte, sino más bien presentarlo como una actividad humana cuya finalidad es otorgar sentido y significado a nuestra existencia, aportando experiencias enriquecedoras a nuestras vidas (DEWEY, 1949; AGIRRE, 2005).

Después de esta sesión, se propuso a los estudiantes la realización de un trabajo que sintetizara sus experiencias entorno a la práctica de la pintura y que fuera concebido como un instrumento de comunicación y expresión. Se les propuso realizar "un libro de artista" a través del cual debían reflejar y materializar todos aquellos elementos que configuraban su universo particular, para ello se les explicó que era un "libro de artista", y se les mostraron diversos ejemplares a través de imágenes. 
Conscientes de que la propuesta era compleja, dada la escasa formación artística que reciben los estudiantes durante la etapa de educación obligatoria, el trabajo se planteó como una forma de cristalizar sus experiencias entorno a la pintura, y de recrear una situación que les obligara a explorar las posibilidades de la práctica artística como un sistema simbólico donde evocar significados. (COLLADOS y ROSICH, 2008)

\section{SEGUNDO PROYECTO: “CREATIVIDAD Y COOPERACIÓN”}

“Un caso práctico de aprendizaje por problemas (PBL) aplicado al diseño gráfico."

Este proyecto se llevo a cabo en las aulas del tercer curso de Educación Secundaria Obligatoria, correspondiente a la edad de 14 años.

El proyecto que se decidió desarrollar partía de dos decisiones previas: probar la eficacia de la metodología PBL y hacerlo con la parte del currículo correspondiente al diseño gráfico.

El objetivo fundamental era que los alumnos aprendieran a trabajar de forma cooperativa a través del desarrollo de proyectos artísticos. A nivel disciplinar se les proponía un trabajo relacionado con el diseño gráfico, lo que suponía utilizar una buena parte de los contenidos que habían ido aprendiendo a lo largo de los anteriores cursos, como dibujo, color, utilización de estructuras compositivas, así como incorporar sus conocimientos relacionados con el uso de las tecnologías de la información y comunicación al ámbito artístico.

El proyecto se articuló en dos partes, que aglutinaban dos grandes tareas:

La primera consistía en crear una pequeña empresa y diseñar su imagen corporativa acorde con su filosofía y proyecto empresarial. Para ello era necesario averiguar el funcionamiento de una empresa de diseño, conocer los diferentes cargos y sus funciones, y plantearse su filosofía. A continuación, inventar un nombre, diseñar su imagen corporativa y preparar una presentación para darse a conocer a los potenciales clientes, tarea que daba por concluida esta primera parte, y que significaba su entrada al mercado como empresa.

La segunda tarea, consistió en desarrollar los primeros trabajos que llegaron a la empresa con la máxima diligencia, eficacia y creatividad. Estos trabajos consistieron en el diseño de un cartel que anunciaba una página Web de descarga de música libre, el diseño de una carátula de CD de alguno de los grupos de música incluidos en dicha página Web, y el diseño de una bolsa para transportarlos.

Para aportar más credibilidad a la "situación" que estábamos intentando recrear con la metodología de "Problem Based Learning" PBL, la vinculamos a un proyecto creado por un grupo de jóvenes de su misma edad, y consistente en una página Web (www.musicalliure.cat) donde se podía descargar música de forma libre, gratuita y legal. Este proyecto nacido a partir de la iniciativa de jóvenes cuyas edades oscilaban entre los 15 y los 18 años y sin ningún tipo de financiación, era un buen ejemplo de proyecto creativo y emprendedor que además contaba con bastante repercusión mediática.

Se les propuso que diseñaran un cartel para promocionar esa página, que transmitiera la filosofía del proyecto. Con ello se les planteaba el reto de transformar una idea en una imagen, aplicando todos sus conocimientos y potencial como equipo.

Gracias al contacto con los creadores de la página, conseguimos el compromiso de que los carteles fueran colgados en la página de música y vistos en la red, hecho que incorporó veracidad y estímulo a la situación.

También debían escoger a uno de los cantantes o grupos que libremente ofrecían su música en la red, y crear un diseño de carátula para uno de sus trabajos, así como una pequeña bolsa para transportarlo. Esta segunda parte concluía en una sesión donde se mostraban los resultados obtenidos y se narraba el proceso seguido.

\section{TERCER PROYECTO: “IDENTIDADES”}

Un proyecto que se articula entorno al autorretrato como instrumento de auto conocimiento. 
Este último proyecto se desarrolló en el nivel correspondiente a la edad de catorce años y que coincide con un momento delicado de la vida de los jóvenes: el inicio de la adolescencia, que a menudo provoca dificultades para aceptarse y comprenderse.

A partir de esta idea se articuló una secuencia de aprendizaje que giraba entorno al autorretrato, y que proponía una serie de propuestas artísticas concebidas como parte de un proceso que concluiría en la creación de un autorretrato que cristalizara su forma de mostrarse al mundo a través del lenguaje artístico.

Este proyecto, tenía como principal objetivo promover y desarrollar un proceso creativo que les llevara a reflexionar sobre el tema propuesto y les invitara a pensar a través de la práctica artística.

\section{4- PRESENTACIÓN DE LOS RESULTADOS OBTENIDOS}

De la observación y análisis de la situación de la educación artística en los niveles de educación secundaria obligatoria en relación a como se producen sus aprendizajes, se observa cierto decalaje entre el currículo escrito, representado por las programaciones y los libros de texto, y la práctica que se produce en las aulas, que por motivos diversos no siempre consigue desarrollar las estrategias necesarias para transmitir los objetivos previstos.

En las entrevistas se constató que el actual currículo se aprecia disperso y excesivamente ambicioso, coincidiendo también en afirmar la imposibilidad de desarrollarlo de forma íntegra en el tiempo de que disponen. Ambos hechos favorecen incumplimientos curriculares que se producen acordes a las prioridades que establece el docente y no exentas de ciertos paralelismos con sus fortalezas y debilidades didácticas.

Los libros de texto son reconocidos por los docentes como su referente más directo del currículo oficial, otorgándoles una función relevante en las prácticas docentes que se desarrollan en el aula. El análisis de estos materiales didácticos revela, en una significativa proporción, cierta incoherencia entre una teoría con una elevada presencia de conceptos de considerable complejidad que se pre- sentan con un sofisticado vocabulario y unas prácticas desiguales y a menudo carentes de vinculación directa con sus objetivos didácticos, hechos que no favorecen un aprendizaje significativo, contextualizado y dotado de sentido.

Los docentes coinciden en valorar como insuficiente la formación didáctica recibida en su etapa de de formación inicial, reconociendo también ciertas dificultades en relación a la dinámica del aula o en la forma de abordar su diversidad.

Las observaciones de aula corroboran también que las metodologías docentes no presentan una gran diversificación, siendo la más común un elemental binomio entre la expositiva y la experimental.

En relación al análisis de los resultados obtenidos en el desarrollo de las propuestas didácticas, se observa que las metodologías más activas y las secuencias de aprendizaje más extensas, coherentes y contextualizadas dinamizan los procesos de aprendizaje e implican más a los estudiantes en su proceso de formación, dando más sentido a los aprendizajes e incrementando el grado de satisfacción de docentes y alumnos.

La figura del docente como mediador de los procesos de enseñanza y aprendizaje se configura como la clave de toda acción educativa, su formación es fundamental en la mejora de todos los procesos formativos.

El docente necesita recursos didácticos que orienten y proporcionen soporte a las tareas que implican su intervención curricular. El intercambio de experiencias y el trabajo cooperativo con otros profesionales se revelan como factores que contribuyen a romper el aislamiento profesional y a construir conocimiento profesional válido y útil.

La desconexión entre los niveles educativos de primaria y secundaria, es percibida por los docentes con gran preocupación, ya que se traduce en una base de conocimientos de escasa solidez y repercute de forma negativa en la adquisición y consolidación de buenos niveles de aprendizaje en esta área. 


\section{5-CONCLUSIONES Y PROPUESTAS DE ACTUACIÓN}

De la investigación desarrollada se desprenden las siguientes conclusiones, que apuntan alguno de los factores que podrían incorporar mejoras en la calidad de la educación artística en la etapa 12-16 años.

1- En la educación artística correspondiente a los niveles de Educación Secundaria Obligatoria existe un decalaje entre la teoría representada por el currículo escrito, (programaciones oficiales y libros de texto) y la práctica que se realiza en las aulas, producto de la escasa solidez en las líneas de un discurso-base caracterizado por una gran indefinición.

2- La mejora de la educación artística y de sus procesos de aprendizaje, está vinculada de forma directa a la formación del profesorado, revelándose sus concepciones acerca de aquello que debe ser la educación artística, como una de las piezas clave del proceso. Estas y las de la institución escolar que lo acoge acerca del valor educativo que le otorgan a la práctica del arte se revelan importantes para el óptimo desarrollo de su labor.

3- Se observa una considerable desconexión entre la etapa de primaria, que cubre las edades 6-12 y la de secundaria 12-16, hecho que no contribuye a desarrollar una educación artística coherente y significativa, que sea una herramienta que nos ayude a comprender y a comprendernos.

4- La experimentación curricular realizada en los tres centros participantes apunta que las secuencias de aprendizaje más extensas, junto con la incorporación de metodologías didácticas más activas y participativas, dinamiza e incorpora sentido a los aprendizajes, aumentando el grado de satisfacción de los estudiantes, su autoestima y la percepción positiva de la comunidad escolar.

Las propuestas de actuación que se desprenden del análisis de los resultados son las siguientes:

1- Es necesario definir un modelo decidido de educación artística acorde con los nuevos retos que plantea la sociedad y aunar esfuerzos para hacerlo tangible, desarrollando e identificando prácticas y estrategias eficaces para los objetivos propuestos.
2- Se revela de vital importancia que los diversos agentes educativos que giran entorno al universo escolar (escuela, universidad), incrementen sus relaciones y vinculen los diversos tipos de conocimientos con los que operan, con el objetivo común de construir la clase de conocimiento necesario para ir transformando y mejorando nuestras escuelas.

Para ello es indispensable producir experiencias que aporten significado y pongan a prueba la teoría, impulsando la cultura de la práctica reflexiva y del trabajo cooperativo entre docentes de diversos niveles, para seguir aprendiendo y avanzando juntos.

\section{5-BIBLIOGRAFIA}

AGIRRE, I. (2005) Teorías y prácticas en educación artística. UPN: Octaedro-EUB.

ARAÑÓ, J.C. (1989) La enseñanza de las Bellas Artes como forma de ideología cultural, Arte, individuo y sociedad, 2, 9-30.

ARNHEIM, R. (1993) Consideraciones sobre la educación artística. Barcelona: Paidós.

BENEJAM, P. (2004) De la teoría... a l'aula. Reflexions sobre fer de mestre. Lliçó Magistral: UAB. (Paper)

COCHRAN - SMITH, M. - LYTLE, S.L. (2002) DENTRO/FUERA. Enseñantes que investigan. Madrid: Akal.

COLLADOS, E. - ROSICH, C. (2008) La pintura puede serlo todo, Cuadernos de Pedagogía 378, 31-33.

DEWEY, J. (2004) Experiencia y educación. Madrid: Biblioteca Nueva.

EISNER, E. W. (1995) Educar la visión artística. Barcelona: Paidós.

ESCUDERO, J.M., GÓMEZ, A.L. (ed.) (2006) La formación del profesorado y la mejora de la educación. Barcelona: Octaedro.

GARDNER, H. (1994) Educación artística y desarrollo humano. Barcelona: Paidós.

MARÍN VIADEL, R. (Coord.) (2003) Didáctica de la Educación Artística para Primaria. Madrid: Pearson Educación.

STOLL, FINK D., EALL, L. (2004) Sobre el aprender y el tiempo que requiere; implicaciones para la escuela. Barcelona: Octaedro. 